\title{
FREQÜÊNCIA DA ATIVIDADE SEXUAL EM MULHERES MENOPAUSADAS
}

\author{
Dino Roberto Soares De Lorenzi*, Bruno Saciloto
}

Trabalho realizado na Disciplina de Ginecologia do Curso de Medicina da Universidade de Caxias do Sul, Caxias do Sul, RS

\section{* Correspondência}

Rua Bento Gonçalves, 1759/602

Cep: $95020-412$

Caxias do Sul, RS

\begin{abstract}
RESUMO
Oвjetivo. Identificar os fatores relacionados à freqüência da atividade sexual entre mulheres pós-menopáusicas.

Métodos. Estudo transversal de 206 mulheres pós-menopáusicas entre 45 e 60 anos atendidas em um serviço universitário da região Sul do Brasil entre junho e outubro de 2002. A atividade sexual foi avaliada pelo número de relações sexuais no último mês e a sintomatologia climatérica pelo Índice de Kupperman. Na análise estatística, fez-se regressão linear múltipla. Resultados. Das mulheres pesquisadas, I76 (85\%) eram sexualmente ativas. Cerca de 60,6\% relataram diminuição da atividade sexual após a menopausa, o que atribuíram principalmente à impotência sexual do parceiro (41,7\%). Aproximadamente $25,7 \%$ negaram satisfação com 0 intercurso sexual. Na análise por regressão linear múltipla, associaram-se à atividade sexual a idade $(p<0,01)$, o grau de satisfação sexual $(p=0,01)$ e a sintomatologia climatérica $(p=0,02)$. Quanto maior a idade, mais intensa a sintomatologia climatérica, menor a satisfação sexual e menos freqüente a atividade sexual. Os sintomas climatéricos que se correlacionaram com a atividade sexual foram os fogachos $(p=0,05)$, a irritabilidade $(p=0,04)$, a melancolia/tristeza $(p=0,04)$, as artralgias/mialgias $(p<0,01)$ e a fraqueza/cansaço $(p<0,01)$.

Conclusäa. Os achados deste estudo foram similares aos descritos na literatura. Estes reforçam a hipótese da sexualidade da mulher climatérica não ser influenciada somente por fatores relacionados ao hipoestrogenismo, como também por fatores psicossociais e culturais associados ao próprio envelhecimento. Todavia, estudos Iongitudinais são necessários para se obter dados mais conclusivos. Especial atenção deve ser dada para as disfunções sexuais masculinas.
\end{abstract}

Unitermos: Climatério. Menopausa. Sexualidade feminina.

\section{INTRODUÇÃO}

A sexualidade é reconhecida atualmente como um dos pilares da qualidade de vida, sendo a sua abordagem cada vez mais valorizada! Caracteriza-se pelo caráter multidimensional, ou seja, não é influenciada somente por fatores anatômicos e fisiológicos, como também por fatores psicossociais e culturais, além de relacionamentos interpessoais e experiências de vida?

No climatério, as questões relacionadas à sexualidade têm se revestido de crescente valorização em virtude da crescente longevidade feminina nas últimas décadas e da maior prevalência de disfunções sexuais depois da menopausa $a^{2,3}$. Estudo envolvendo 4753 ginecologistas brasileiros revelou que a diminuição do desejo sexual estava entre os principais motivos de procura por consultas ${ }^{4}$. Enquanto cerca de $25 \%$ a $33 \%$ das mulheres com idade entre 35 e 59 anos manifestam disfunções sexuais, entre 60 e 65 anos estes percentuais variam de $51 \%$ a $75 \%{ }^{5}$.

O impacto do climatério na sexualidade feminina, contudo, não está totalmente esclarecido. Persistem controvérsias sobre a idade como fator mais importante do que o próprio estado menopausal na sexualidade feminina ${ }^{6}$. A queda dos níveis de estrogênio resulta na diminuição do suporte pélvico e da lubrificaçãa dos tecidos urogenitais, causando dispareunia e dificultando a atividade sexual 2,7,8. $^{\circ}$ hipoestrogenismo promove também a redução do colágeno cutâneo e alterações na distribuição de gordura corporal, causando mudanças na configuração corporal, o que, por sua vez, afetaria a auto-imagem feminina, favorecendo uma menor auto-estima e a perda do desejo sexual ${ }^{8}$.

Muitas mulheres percebem a chegada da menopausa como a perda do objetivo primário do sexo, a reprodução, o que pode se refletir no exercício da sexualidade. Assim, eventuais sentimentos de culpa em relação aos impulsos sexuais poderiam explicar a maior prevalência de disfunções sexuais no período pós-menopausa ${ }^{8,9}$.

No Brasil, a despeito da relevância do tema, são ainda escassos os estudos voltados para as questões relacionadas à sexualidade no climatério, o que poderia contribuir para o planejamento da assistência à mulher no seu processo de envelhecimento. Este estudo teve por objetivo identificar possíveis fatores preditores da freqüência da atividade sexual em um grupo de mulheres climatéricas atendidas em um serviço universitário.

\section{Métodos}

Foram entrevistadas 206 mulheres pós-menopáusicas com idade entre 45 e 60 anos atendidas no Ambulatório de Climatério da Universidade de Caxias do Sul, RS, entre junho e outubro de 2002.

O estado menopausal foi definido segundo a história menstrual, tendo sido considerada pós-menopáusica toda mulher cujo último fluxo menstrual espontâneo ocorreu havia pelo menos 12 meses ${ }^{1,3,6}$. Devido à dificuldade de estabelecer clinicamente o seu estado menopausal, excluiu-se as mulheres com histerectomia prévia. 
As variáveis estudadas incluíram: idade, renda familiar per capita em salários mínimos (total dos rendimentos dos indivíduos da família dividido pelo número total de pessoas que a integram), escolaridade (total de anos completos de estudo), cor (esta definida pela própria paciente como branca ou não branca), ocupação remunerada (dicotomizada em sim ou não), número de gestações prévias, sexarca (idade da primeira relação sexual), atividade sexual (número de relações sexuais no último mês), satisfação sexual, idade da menopausa (última menstruação seguida de 12 meses de amenorréia), história de tabagismo no último ano (dicotomizada em sim ou não), índice de massa corporal (IMC) e a história de comorbidades clínicas (dicotomizada em sim ou não).

A satisfação sexual foi avaliada por uma escala de três pontos ("sempre", "freqüentemente" ou "nunca"). Quanto maior a pontuação, maior a satisfação sexual.

A sintomatologia climatérica foi avaliada pelo Índice de Kupperman (IK). Este é composto de II sintomas ou queixas (sintomas vasomotores, insônia, parestesia, nervosismo, melancolia, vertigem, fraqueza, artralgia/mialgia, cefaléia, palpitação e zumbidos), os quais recebem diferentes pontuações segundo a sua intensidade e prevalência, sendo classificados em leves (até 19 pontos), moderados (entre 20 e 35 pontos) ou severos (mais de 35 pontos) ${ }^{10}$.

Os dados obtidos foram analisados por meio do programa Statistical Package for Social Sciences for Personal Computer (SPSS-PC) versão 12 , considerando-se um nível de significância de 5\%.

Inicialmente, foram descritas as freqüências simples, médias e desvio padrão. Para a análise de diferenças de médias, utilizou-se o teste $t$ de Student e a análise de variância (Anova). A avaliação da associação entre as variáveis estudadas e o desfecho (número de relações sexuais referido no último mês) foi possível por meio de regressão linear múltipla (método Enter). Para viabilizar a sua inclusão no modelo de regressão, as variáveis categóricas foram transformadas em variáveis "dummy".

Este estudo foi previamente aprovado pelo Comitê de Ética em Pesquisa da Universidade de Caxias do Sul. Antes da inclusão de qualquer paciente, esta foi esclarecida acerca da sua metodologia, objetivos e riscos, assinando um termo de consentimento livre e esclarecido.

\section{Resultados}

Das 206 pacientes avaliadas, 175 eram sexualmente ativas ( $85 \%$ ), com uma idade média de 53,5 anos $( \pm 4)$ anos. Entre as mulheres sem atividade sexual ( $\mid 5 \% / n=3 I)$, a idade média observada foi $54,2( \pm 4, I)$ anos.

A maioria das entrevistadas $(60,6 \% / n=106)$ referiu diminuição da freqüência das relações sexuais após a menopausa (Tabela I), com uma média de $6,3( \pm 3,1)$ relações sexuais no último mês. A principal causa de diminuição da atividade sexual foi a impotência sexual do parceiro $(41,7 \%)$.

Cerca de 80,6\% tinham entre 50 e 60 anos de idade (80,6\%), $70,3 \%$ eram brancas e $52,6 \%$ referiram menos de cinco anos completos de estudo. Ocupação remunerada foi confirmada por 33,7\% das entrevistadas, com uma renda familiar inferior a um salário mínimo per capita em 46,9\% dos casos (Tabela 2).

A menarca ocorreu em média aos 13,3 $( \pm 1,8)$ anos, ao passo que a sexarca (primeira relação sexual) aos 20,9 $( \pm 5,4)$ anos. A idade média

\begin{tabular}{lccc}
\hline \multicolumn{3}{c}{ Tabela I - Causas de diminuição da frequiência da atividade sexual } \\
\hline Causas & \multicolumn{1}{c}{$\mathbf{n}^{*}$} & & $\%$ \\
\cline { 2 - 4 } Ausência de um companheiro fixo com quem se relacionar & & & 6,8 \\
Dispareunia & 21 & & 12,0 \\
Díficuldades no relacionamento com o parceiro & 28 & & 16,0 \\
Doenças ou problemas clínicos da própria mulher & 34 & 19,4 \\
Falta de desejo sexual & 36 & 20,6 \\
Impotência sexual do parceiro & 73 & 41,7 \\
\hline
\end{tabular}

* Uma das causas pode ter sido referida por mais de uma das mulheres entrevistadas

da menopausa foi $47,9( \pm 4,1)$ anos. $O$ número médio de gestações prévias foi $3,3( \pm 1,6)$, sendo que $5,7 \%$ das mulheres estudadas nunca engravidaram.

Quanto às condições de saúde, 21,7\% das pacientes eram tabagistas, apresentando uma prevalência de sobrepeso ou obesidade (IMC $\left.\geq 25 \mathrm{Kg} / \mathrm{m}^{2}\right)$ de 33,7\%. Comorbidades clínicas foram referidas por $67,4 \%$ das mulheres pesquisadas, entre as quais a hipertensão arterial $(51 \% / n=105)$, o diabetes $(16 \% / n=33)$, o infarto agudo do miocárdio $(5,8 \% / n=12)$ e o acidente vascular cerebral $(5,3 \% / n=\mid I)$.

Terapia hormonal no último ano foi confirmada por $22,9 \%$ das entrevistadas. Em relação à sintomatologia climatérica (Índice de Kupperman), esta se mostrou leve em $28 \%$, moderada em $45,1 \%$ e severa em $26,9 \%$.

A freqüência das relações sexuais (Tabela 2 ) associou-se significativamente com a idade $(p=0,02)$, o nível de satisfação sexual $(p \leq 0,0$ I $)$ e a intensidade dos sintomas climatéricos $(p=0,02)$. O número médio de relações sexuais foi significativamente menor entre as mulheres com mais de 50 anos e com menor satisfação sexual.

Cerca de $25,7 \%$ das entrevistadas negaram prazer ou satisfação sexual. Conforme mostra a Tabela 2, as relações sexuais foram menos freqüentes entre as mulheres que negaram prazer sexual, quando comparadas com as que referiram ter sempre prazer $(p=0,02)$ ou pelo menos freqüentemente $(p<0, I)$, não tendo sido identificadas diferenças significativas entre estes dois últimos grupos $(p=0, \mid 3)$.

A sintomatologia climatérica se correlacionou com a freqüência da atividade sexual $(p=0,05)$. Quanto maior o escore do Índice de Kupperman (IK), menor a atividade sexual (Tabela 3). Os sintomas que compõem o IK que se correlacionaram com a frequêencia das relações sexuais foram a irritabilidade $(p=0,04)$, a melancolia/tristeza $(p=0,04)$, as artralgias/mialgias $(p<0,0 \mathrm{I})$, os fogachos $(p=0,05)$ e a fraqueza/cansaço $(p<0,0 \mathrm{I})$.

$A$ análise por regressão linear múltipla, tendo como variável dependente o número de relações sexuais no último mês (Tabela 4), revelou como preditores da frequêencia da atividade sexual as seguintes variáveis: idade $(p<0,01)$, intensidade da sintomatologia climatérica $(p=0,02)$ e nível de satisfação com a atividade sexual $(p=0,01)$.

\section{Discussão}

O climatério tem sido descrito como a transição entre a fase reprodutiva e não reprodutiva da vida da mulher, que, além de oscilações hormonais e alterações estéticas, envolve mudanças psicológicas e no seu papel social, o que se reflete na esfera sexual?. 
Tabela 2 - Freqüência média de relações sexuais segundo variáveis sociodemográficas, reprodutivas e condiçōes de saúde

\begin{tabular}{|c|c|c|c|}
\hline Variáveis & $n(\%)$ & $\begin{array}{l}\text { Número de relações } \\
\text { sexuais Média (DP) }\end{array}$ & $\mathbf{p}^{*}$ \\
\hline \multicolumn{4}{|l|}{ Idade (em anos) } \\
\hline $45-49$ & $34(19,4)$ & $8,5(5,9)$ & \\
\hline $50-60$ & $|4|(80,6)$ & $5,8(4,9)$ & 0,02 \\
\hline Média(DP) & $53,5( \pm 4,0)$ & & \\
\hline \multicolumn{4}{|l|}{ Cor } \\
\hline Branca & $123(70,3)$ & $6,1(5,1)$ & \\
\hline Não branca & $52(29,7)$ & $6,5(4,8)$ & 0,75 \\
\hline \multicolumn{4}{|c|}{ Anos completos de estudo } \\
\hline$<5$ & $92(52,6)$ & $5,9(5,4)$ & \\
\hline$\geq 5$ & $83(47,4)$ & $6,7(5,0)$ & 0,36 \\
\hline Média(DP) & $5, \mid(\mid 3,8)$ & & \\
\hline \multicolumn{4}{|c|}{ Ocupação remunerada } \\
\hline Sim & $59(33,7)$ & $6,4(5,6)$ & \\
\hline Não & $116(66,3)$ & $6,3(4,5)$ & 0,94 \\
\hline \multicolumn{4}{|c|}{ Renda familiar per capita } \\
\hline$<$ | salário mínimo & $82(46,9)$ & $6,3(5,5)$ & \\
\hline 2 | salário mínimo & $93(53,1)$ & $5,9(4,9)$ & 0,99 \\
\hline Média(DP) & $1,5(2,8)$ & & \\
\hline \multicolumn{4}{|l|}{ História obstétrica } \\
\hline Nunca engravidaram & $10(5,7)$ & $4,1(4,3)$ & \\
\hline Com gestações prévias & $165(94,3)$ & $6,5(5,2)$ & 0,06 \\
\hline \multicolumn{4}{|c|}{ Prazer na atividade sexual } \\
\hline Sempre & $58(33,1)$ & $7,9(5,1)$ & \\
\hline Freqüentemente & $72(41,1)$ & $6,1(4,1)$ & $<0,0 \mid * *$ \\
\hline Nunca & $45(25,7)$ & $4,5(2,4)$ & \\
\hline \multicolumn{4}{|l|}{ Idade da menopausa } \\
\hline$<50$ anos & $107(61,1)$ & $6,4(4,4)$ & \\
\hline$\geq 50$ anos & $68(38,9)$ & $6,2(3,9)$ & 0,75 \\
\hline Média(DP) & $47,9(4,4)$ & & \\
\hline \multicolumn{4}{|l|}{ Tabagismo } \\
\hline Sim & $38(21,7)$ & $5,7(4,8)$ & \\
\hline Não & $137(78,3)$ & $6,5(5,2)$ & 0,38 \\
\hline \multicolumn{4}{|c|}{ Índice de massa corporal } \\
\hline$\geq 25 \mathrm{~kg} / \mathrm{m}^{2}$ & $116(66,3)$ & $6,7(5,5)$ & \\
\hline$<25 \mathrm{~kg} / \mathrm{m}^{2}$ & $59(33,7)$ & $6,1(5,1)$ & 0,42 \\
\hline Média(DP) & $27,8(5,3)$ & & \\
\hline \multicolumn{4}{|l|}{ Terapiahormonal } \\
\hline $\operatorname{Sim}$ & $40(22,9)$ & $7,8(6,0)$ & \\
\hline Não & $135(77,1)$ & $5,8(4,9)$ & 0,08 \\
\hline \multicolumn{4}{|c|}{ Comorbidades clínicas } \\
\hline Sim & $1 \mid 8(67,4)$ & $6,7(5, I)$ & \\
\hline Não & $57(32,6)$ & $6,1(5,7)$ & 0,48 \\
\hline Total & $175(100)$ & & \\
\hline
\end{tabular}

*Teste t de Student

**Anova
Tabela 3 - Correlação entre os sintomas climatéricos e a freqüência da atividade sexual

\begin{tabular}{lcc}
\hline Sintomas & $\mathbf{r}^{*}$ & $\mathbf{p}$ \\
\cline { 1 - 1 } Irritabilidade & $-0,15$ & 0,04 \\
Artralgia/mialgia & $-0,20$ & $<0,01$ \\
Melancolia/tristeza &,- 016 & 0,04 \\
Zumbidos & $-0,11$ & 0,07 \\
Insônia & $-0,09$ & 0,20 \\
Parestesias & $-0,01$ & 0,90 \\
Cefaléila & $-0,08$ & 0,31 \\
Fogachos & $-0,12$ & 0,05 \\
Vertigem & $-0,12$ & 0,11 \\
Fraqueza/cansaço & $-0,21$ & $<0,01$ \\
Índice de Kupperman total & $-0,20$ & 0,05 \\
\hline * Coeficiente de correlação de Pearson & &
\end{tabular}

\begin{tabular}{lcc}
\hline $\begin{array}{c}\text { Tabela } 4 \text { - Fatores preditores da freqüência da atividade sexual no período } \\
\text { pós-menopausa: análise por regressão linear múltipla }\end{array}$ \\
\hline Variáveis* & $\boldsymbol{\beta}$ & $\boldsymbol{p}$ \\
Idade (em anos) & $-0,23$ & $\frac{\boldsymbol{p}}{<0,01}$ \\
Prazer nas relaçōes sexuais & 0,18 & 0,02 \\
Intensidade da sintomatologia climatérica & $-0,20$ & 0,01 \\
\hline
\end{tabular}

*Análise ajustada para: idade, escolaridade, cor, ocupação, renda familiar, sexarca, número de gestações, idade da menopausa, terapia hormonal, tabagismo, índice de massa corporal e intensidade da sintomatologia climatérica

A maioria das mulheres estudadas referiu diminuição da atividade sexual após a menopausa, achado este corroborado por outros pesquisadores. Estudo similar realizado no Chile revelou que $74 \%$ das mulheres entre 40 e 44 anos são sexualmente ativas, com uma média mensal de seis relações sexuais, enquanto que, entre os 55 e 59 anos, somente $40 \%$ são sexualmente ativas, com uma média mensal de 2,7 relações sexuais". No Brasil, pesquisa envolvendo 400 mulheres usuárias do Setor de Climatério da Universidade Federal de São Paulo revelou que $82,7 \%$ destas eram sexualmente ativas, com uma média de mensal de três relações sexuais ${ }^{12}$.

Para Dennerstein et al. (200 I), a diminuição da libido e da freqüência das relações sexuais no climatério pós-menopáusico estariam associadas principalmente à maior prevalência de dispareunia e fogachos nesse período?. Entre as causas do decréscimo da atividade sexual no climatério, estão a maior ocorrência de dispareunia decorrente de atrofia urogenital e a diminuição do desejo sexual ".

Para De Paula (2002), 86,5\% das queixas relacionadas à sexualidade no climatério ocorrem na pós-menopausa (86,5\%), entre estas a dispareunia e a diminuição do desejo sexual' ${ }^{12}$. Ortiz e Corona (2000), ao investigarem o perfil sexual de 100 mulheres climatéricas e seus parceiros, constataram uma diminuição da freqüência das relações sexuais, cujos motivos incluíram a ocorrência de dispareunia e a redução do desejo sexual|' . Flores et al. ( 1998), por sua vez, investigaram a sexualidade de 106 mulheres climatéricas, verificando uma redução da freqüência sexual de 60\%, decorrente principalmente da redução do desejo sexual|' . 
A redução da atividade sexual entre as mulheres pesquisadas foi atribuída também a eventuais dificuldades no relacionamento conjugal ou à falta de um companheiro fixo com quem se relacionar, conforme descrito por outros autores ${ }^{15}$.

Para Avis et al. (2000), o estado menopausal estaria envolvido principalmente com a ocorrência de dispareunia. Outros demais fatores interfeririam igualmente na sexualidade da mulher climatérica, entre estes a idade, as suas condições de saúde, o seu estado marital, a história de tabagismo e eventuais fatores psicossociais ${ }^{16}$.

Danaci et al. (2003) atribuíram a diminuição da freqüência de relações sexuais no climatério à maior ocorrência de estados depressivos ou de maior ansiedade nesse período ${ }^{17}$. No imaginário feminino, a menopausa representaria o envelhecimento, maior proximidade da morte e decadência sexual, favorecendo estados depressivos e uma menor atividade sexual18.

A influência da terapia hormonal na sexualidade da mulher climatérica tem se mostrado também controversa". Em nosso estudo, ao contrário do observado por Blummel et al. (2003) ${ }^{19}$, a terapia hormonal não se associou à atividade sexual, ainda que melhore o trofismo urogenital e reduza os sintomas vasomotores, - que reforça a teoria de que a sexualidade no climatério não é influenciada somente por fatores hormonais, mas também por fatores psicossociais ${ }^{8}$.

Entre as mulheres estudadas, a freqüência sexual correlacionou-se significativamente com a ocorrência de melancolia, tristeza e ansiedade. Para Favarato et al. (2000), as reações emocionais no climatério são inúmeras e variáveis. Entre as mulheres que vivenciam positivamente essa fase, as repercussões sexuais são menos intensas, pois percebem na redução das obrigações com os filhos e com a profissão uma oportunidade para o exercício afetivo-sexual. Já para as mulheres que associam a perda da capacidade reprodutiva à velhice, a sexualidade pode ser muito comprometida. O próprio envelhecimento físico pode comprometer a auto-estima feminina, em especial nas culturas ocidentais, em que predomina uma visão estereotipada e negativa acerca do envelhecimento feminino? ${ }^{2}$.

A maior prevalência de melancolia e tristeza no climatério tem sido associada a transtornos depressivos prévios, a dificuldades como o sono e a ondas de calor comuns nessa fase. O próprio hipoestrogenismo causa redução da secreção de endorfinas cerebrais, favorecendo a depressão ${ }^{20}$. Além disso, a diminuiçãa dos níveis de testosterona observada em algumas mulheres contribuiria para a diminuição da atividade sexual e da libido'21.

Apesar da tendência de diminuição da atividade sexual nos anos que se seguem à menopausa, o interesse sexual parecer nem sempre se alterar. Segundo McCoy et al. (1985), nos anos que antecedem a menopausa, é comum o declínio do interesse sexual e das fantasias sexuais, no entanto, a freqüência orgástica tende a se manter sem alterações ${ }^{22}$. No corrente estudo, $74,2 \%$ das mulheres entrevistadas referiram ter sempre prazer nas relações sexuais ou pelo menos freqüentemente. Cerca de 6,8\% e 41,7\% das entrevistadas, respectivamente, justificaram a diminuição da atividade sexual pela falta de um companheiro fixo e a ocorrência de impotência sexual do parceiro.

É necessário lembrar que, durante o envelhecimento masculino, há uma queda progressiva da secreção de testosterona, favorecendo a ocorrência de disfunções sexuais, entre estas a diminuição da libido masculina, transtornos de ereção e alterações espermáticas ${ }^{21}$. Levantamento realizado no Chile revelou um percentual de transtornos de ereção ao redor de $55 \%$ entre os parceiros de 100 mulheres climatéricas ${ }^{13}$.

Além disso, após os 60 anos, a prevalência de neoplasias de próstata é significativamente maior, sendo que seu tratamento cirúrgico pode vir a comprometer a capacidade de ereção ${ }^{2 !}$.

No climatério, a freqüência das relações sexuais seria influenciada pelo próprio processo de envelhecimento, este manifestado pela principalmente pela idade conforme observado em no presente estudo?. O envelhecimento feminino é acompanhado por uma maior ocorrência de humor depressivo, ansiedade, irritabilidade e sintomas vasomotores que, somados a fatores psicossociais e culturais, podem interferir na atividade sexual ${ }^{16}$

A maior prevalência de disfunções sexuais no climatério reforça a necessidade dos ginecologistas voltarem a sua atenção para a ocorrência de dificuldades na esfera sexual a cada consulta médica. No entanto, é reconhecido que estes, não raramente, encontram-se pouco preparados para abordar eventuais dificuldades relacionadas à sexualidade feminina, sobretudo entre as mulheres mais idosas ${ }^{23}$.

\section{Conclusão}

O conhecimento das necessidades sexuais e dificuldades femininas no climatério é fundamental para a promoção da qualidade de vida da mulher no seu processo de envelhecimento. É importante que as mulheres climatéricas, assim como seus parceiros, sejam informados sobre as mudanças orgânicas e de comportamento a que estão sujeitos durante o envelhecimento, o que certamente facilitará a identificação de eventuais dificuldades na esfera sexual e as intervenções terapêuticas mais indicadas.

\section{Conflito de interesse: não há}

\section{SUMMARY}

\section{FACTORS RELATED TO FREQUENCY OF SEXUAL ACTIVITY OF POSTMENOPAUSAL WOMEN}

OBIECTIVE. To identify factors related to the frequency ofsexual activity ofpostmenopausalwomen

Methods. A cross-section study of 206 postmenopausal women between 45 and 60 years of age was made at a university health care service in the South of Brazil between June and October 2002. Evaluations were made of sexual activity according to the number of sexual intercourses in the previous month and the climacteric symptoms using the Kupperman index. Statistical analysis was performed with multiple linear regressionanalysis.

RESULTS. Of those surveyed I 76 (85\%) women were sexually active. Although $60.6 \%$ reported a decrease in sexual activity after menopause, mostly attributing it to the husband's sexual impotence (41.7\%). Approximately $25.7 \%$ stated they had no satisfaction with sexual intercourse. By means of multiple linear regression analysis the following aspects were associated to sexual activity: age $(p<0.1)$, degree of sexual satisfaction $(p=0.01)$, and climacteric symptomatology $(p=0.02)$. As age 


\section{De LORENZI DRS et AL.}

increased the climacteric symptoms were more intense and sexual activity was less frequent with lower sexual satisfaction. The climacteric symptoms correlated with sexual activity were: hot flashes $(p=0.05)$, irritability $(p=0.04)$, melancholy/sadness $(p=0.04)$, arthralgia/myalgia $(p<0.01)$ and weakness/tiredness $(p<0.01)$.

CONCLUSIONS. Findings of this study were similar to those in literature. They agree with the hypothesis that sexuality of climacteric women is not only influenced by factors related to hypoestrogenism, but also by psychosocial and cultural aspects associated with aging itself. Nevertheless, longitudinal studies are necessary to provide more conclusive data. Special attention should be given to the sexual dysfunction of men. [Rev Assoc Med Bras 2006; 52(4): 256-60]

KEY wORDs: Climacterium. Menopause. Female sexuality.

\section{REFERÊNCIAS}

I. Lopes GP. Sexualidade: fisiopatologia, diagnóstico e tratamento. In: Fernandes CE. Menopausa e tratamento. São Paulo: Editora Segmento; 2003. p.II7-24.

2. Favarato MECS, Aldrighi JM, Fráguas Jr R, Pires ALR, Lima SMRR. Sexualidade e climatério: influência de fatores biológicos, psicológicos e sócio-culturais. Reprod Clim 2000; I 5(4): 199-202.

3. Renó Jr J. Alterações do humor e da cognição: fisiopatologia, diagnóstico e tratamento. In: Fernandes CE, editor. Menopausa e tratamento. São Paulo: Editora Segmento; 2003. p. I I I-6.

4. Abdo $\mathrm{CHN}$, Oliveira Jr WM. O ginecologista brasileiro frente às queixas sexuais femininas: um estudo preliminar. Rev Bras Med 2002;59(3): I 79-86.

5. Blummel JEM, Binfa EL, Cataldo PA, Carrasco AV, Izaguirre HL, Sarrá SC. Índice de función sexual femenina: un teste para evaluar la sexualidad de la mujer. Rev Chil Obstet Gynecol 2004;69(2): I I 8-25.

6. Bossemeyer RP. Aspectos gerais do climatério. In: Fernandes CE, Melo NR, Wehba S. Climatério feminino: fisiopatologia, diagnóstico e tratamento. São Paulo: Lemos; 1999. p. 17-33.

7. Cawood EHH, Bancroft J. Steroid hormones, the menopause, sexuality and well-being of women. Psychol Med 1996;26(4):925-36.

8. Chiechi LM, Granieri M, Lobascio A, Ferreri R, Loizzi P. Sexuality in the climacterium. Clin Exp Obstet Gynecol 1997;24(3): 158-9.
9. Dennerstein L, Dudley E, Burger $\mathrm{H}$. Are changes in sexual functioning during midlife due to aging or menopause? Fertil Steril 200 I;76(4):456-60.

I0. Sousa RL, Sousa ESS, Silva JCB. Fidedignidade do teste-reteste na aplicação do Índice Menopausal de Blatt e Kupperman. Rev Bras Ginecol Obstet 2000, 22(8):48I-7.

I I. Santos MDS, Sala MG. Climatério y menopausia. Parte II. La sexualidad em el climatério. Sexol Soc 1999;5(3): 17-9.

12. De Paula, FJF, Baracat EC, Haidar MA, Lima JGR, Zanetti A, Simões RD. Disfunção sexual no climatério. Femina 2002;30(6):373-6.

13. Damaso MO, Ortigosa EC. Perfil de las relaciones sexuales y sus condiciones em el climaterio. Perinatol Reprod Hum 2000; I 4(3): I 60-7.

14. Flores, DEF, Prieto AFR, Robles VRR, Fierri L, Ruiz A, Martín M. Sexuality and menopause. Ginecol Obstet 1998;44(3):231-8.

15. Bachmann GA, Leiblun SR. The impact of hormones on menopausal sexuality: a literature review. Menopause 2004; I I ( I): I 20-30.

16. Avis NE, Stellato R, Crawford S, Johannes C, Longcope. Is there an association between menopause status and sexual functioning? Menopause 2000;7(5):297-309.

17. Danaci AE, Oruç S, Adigüzel H, Yildirim Y. Relationships of sexuality with psychological and hormonal features in the menopausal period. West Indian Med J 2003;52(I):27-30.

18. Gutiérrez EF. Mas allá del climaterio... Nuestra erótica sexualidad. Rev Colomb Menopaus 200 I;7(3):291-6.

19. Blummel MJE, Bravo MF, Recavarren MA, Salvador CS. Función sexual en mujeres usuarias de terapia de reemplazo hormonal. Rev Med Chile 2003; |3|(2): |25|-5.

20. Aldrighi JM, Aldrighi CMS, Aldrighi APS. Alterações sistêmicas do climatério. Rev Bras Med 2002; 15(1):21.

21. Bulcão CB, Carange E, Carvalho HP, Ferreira-França JB, KligermanAntunes J, Backkes J, et al. Aspectos fisiológicos, cognitivos e psicossociais da senescência sexual. Ciências Cognição 2004; I ( ):54-75

22. McCoy NL, Davidson JM. A longitudinal study of the effects of menopause on sexuality. Maturitas 1985;7(2):203- 10.

23. Penteado SRL, Fonseca AM, Assis JS, Bagnoli VR, Abdo CHN. Sexualidade no climatério e na senilidade. Rev Ginecol Obstet 2002; I I (3): I 88-92.

Artigo recebido: $01 / 04 / 05$

Aceito para publicação: 03/I I/05 